\title{
Inherently fluorescent polyaniline nanoparticles in a dynamic landscape
}

\author{
Clelia Dispenza a,b,*, Maria Antonietta Sabatino ${ }^{a}$, Dagmara Chmielewska ${ }^{\mathrm{a}, 1}$, Caterina LoPresti $^{\mathrm{a}, 2}$, \\ Giuseppe Battaglia ${ }^{c}$
}

a Dipartimento di Ingegneria Chimica, Gestionale, Informatica, Meccanica, Università degli Studi di Palermo, Viale delle Scienze, 90128 Palermo, Italy

${ }^{\mathrm{b}}$ CNR - Istituto di Biofisica (Palermo Unit), Via U. La Malfa 153, 90146 Palermo, Italy

${ }^{\mathrm{c}}$ Department of Biomedical Science, The University of Sheffield, Firth Court, S10 2TN Sheffield, United Kingdom

\section{A R T I C L E I N F O}

\section{Article history:}

Received 13 September 2011

Received in revised form 15 December 2011

Accepted 5 January 2012

Available online 13 January 2012

\section{Keywords:}

Conjugated polymers

Polyaniline

Nanoparticles

Dispersion polymerization

Photoluminescence

\begin{abstract}
A B S T R A C T
In this paper we report for the first time on the emissive behavior of two polyaniline (PANI) nanoparticle systems produced via oxidative chemical polymerization in the presence of either poly(vinyl alcohol) (PVA) or chitosan as polymeric stabilizers in water. The emission from PANI nanoparticles is irreversibly quenched by an increase of $\mathrm{pH}$ of the suspending medium from acid to neutral (chitosan-PANI) or alkaline (PVA-PANI). Conversely, PANI nanorods synthesized in the same conditions of the above, but in presence of poly(N-vinyl pyrrolidone), is not emissive at any $\mathrm{pH}$. The role of the polymeric surfactant as a soft template is key in controlling the morphology and the properties of the obtained PANI dispersions. FTIR, UV-Vis absorption and photoluminescence excitation (PLE) spectra studies suggest that the emissive properties are related to the establishment of strong, non-covalent interactions between nanoscalar PANI particles and the polymeric surfactant at the $\mathrm{pH}$ of synthesis. Morphology examination of the three systems, by both dynamic light scattering (DLS) and Transmission Electron Microscopy (TEM), reveal that photoluminescence is associated to the presence of a genuinely 3D nanoscalar morphology, together with an ordered disposition of PANI chains into aligned crystal planes. Concomitant to the irreversible quenching of the emission signal with increasing $\mathrm{pH}$, there is an evolution of the morphology leading to particle coalescence, coarsening and ultimately phase-separation, with consequent modification of PANI-polymeric surfactant interactions, PANI chains supra-molecular organization and optical properties of the PANI nanoparticles dispersion.
\end{abstract}

(C) 2012 Elsevier Ltd. All rights reserved.

\section{Introduction}

Polyaniline (PANI) identifies a rich family of electronically active, mutually converting polymers $[1,2]$. The free doublet electrons on nitrogen atoms can participate in the formation of the orbitals delocalized along the polymer chain, that determine the semiconducting behavior [3]. For this structural feature, at a difference with other conducting polymers, PANI can undergo to reversible semiconducting to conducting transition by dopingdedoping processes governed by the protonation of its acid groups. These tunable electronic properties together with the environmental stability, relative ease of synthesis and low cost, makes polyaniline is the subject of extensive investigations for applications in

\footnotetext{
* Corresponding author at: Dipartimento di Ingegneria Chimica, Gestionale, Informatica, Meccanica, Università degli Studi di Palermo, Viale delle Scienze, 90128 Palermo, Italy.

E-mail address: clelia.dispenza@unipa.it (C. Dispenza).

1 Present address: Institute of Nuclear Chemistry and Technology, Dorodna 16, 03-195 Warsaw, Poland.

2 Present address: Merck Serono SpA, Via Einaudi 11, 00012 Guidonia Montecelio, Rome, Italy.
}

electronics, industry and medicine [4-8]. Significant differences in electrical and optical properties are reported depending on how polyaniline has been synthesized and/or processed after synthesis. Polyaniline in the conductive emeraldine salt form (PANIES) presents in general less than $1 \%$ of the available charge carriers actually contributing to the intrinsic conductivity of the material [9]. Dangling bonds, chain kinks and other structural defects are responsible for forbidden states in the middle of the conduction band. Accordingly, when polyaniline has been prepared using a self-stabilized dispersion polymerization, that minimize sidebranching (ortho-coupling) and crosslinking, samples with roomtemperature conductivities in excess of $1000 \mathrm{~S} \mathrm{~cm}^{-1}$ and true metallic behavior have been reported [10]. This behavior is associated to minimum disorder-induced localization of the charge carriers and low density of structural defects.

Whereas the electrical conductivity of the different polyaniline variants has been and still is the subject of extensive investigation, through both fundamental and applied research studies, the photoluminescence properties of this family of materials is far less investigated [11-18]. Polyaniline exhibits photoluminescence only its most unstable, fully reduced form of leucoemeraldine [11,12]. This 
is due to the $\pi-\pi *$ transition of the benzenoid units, while quinonoid units act as excitation traps, quenching the photoluminescence produced by the benzenoid units, via intra-chain energy dissipation [13]. Emission of white light from polyaniline, cast into films from solutions, has been observed for PANI both in the emeraldine base and the leucoemeraldine base states, when they were used as the emitting layer of a light emitting diode $[13,14]$. The "exceptional" behavior of the PANI-EB films was attributed to the presence of a "phase segregated" structure in the film, with regions where only oxidized quinoid units or only reduced benzenoid units were concentrated [1,13]. Evidence of photo-luminescence from the emeraldine salt was reported by Gong et al. [14,15]. They show that PANI-ES exhibits fluorescence when the polymer is produced at low temperature through a solid-state synthesis method using an heteropoly acid (a solid-state acid). The authors justify this behavior as a result of a high degree of order in the macromolecular chains orientation, thus providing rigidity and co-planarity. An higher extent of $\pi$-conjugation, coupled with an ordered arrangements of the benzenoid and quinoid units may favor the formation of singlet excitons, that decay to the ground state with emission of light [19].

To the best of our knowledge there is no evidence of photoemission from colloidal PANI. We have already reported on the synthesis of PANI-ES nanoparticles following a chemical oxidative dispersion polymerization route in the presence of either poly $(\mathrm{N}-$ vinyl pyrrolidone) or poly(vinyl alcohol) as steric stabilizers. This was the first of two reactive steps, the second being gamma irradiation of the stabilized PANI dispersion to crosslink the polymeric surfactant, yielding to either PANI-PVP hydrogel or PANI-PVA hydrogel nanocomposites with interesting properties in terms of electrochemical activity and conductivity [20-22]. Photoemission was observed for the dispersions and hydrogels produced with PVA only and at acid $\mathrm{pH}$, although PANI was observed to be in form of emeraldine salt. The unique characteristics of these hydrogel nanocomposites offer applications in many biotechnological areas and, in particular, in (bio)sensing [10]. The potential in applications prompted us to undertake a deeper investigation on the origin of the emissive behavior. In particular, we report here for the first time photoemission also for colloidal protonated emeraldine produced in the presence of chitosan (CT). We provide also an overview of the structural (FTIR), thermogravimetric (TGA), morphological (DLS and TEM), electrical (IS) and optical characterization (UV-Vis absorption, emission and PLE spectroscopy) of both the emitting (PVA-PANI and CT-PANI) and non-emitting (PVP-PANI) colloidal polyanilines, all produced in the same reaction conditions, and investigated at the variance of $\mathrm{pH}$ of the suspending medium, with some additional experimental results as needed.

\section{Experimental}

\subsection{Materials}

Poly(N-vinyl-2-pyrrolidone) (PVP, Mw $=420,000$ from laser light scattering measurements) was supplied as $45 \mathrm{wt} . \%$ aqueous solution by Fluka; poly(vinyl alcohol) (PVA, atatic, $\mathrm{Mw}=47,000$, $88 \%$ degree of hydrolysis) and chitosan (CT, ( $1 \rightarrow 4)$-linked-2-acetamido-2-deoxy- $\beta$-D-glucan (GlcNAc) groups and 2-amino2-deoxy- $\beta$-D-glucan (GlcN) groups randomly distributed along the polymer backbone, $\mathrm{Mv}=50,000-190,000,15-25 \%$ degree of acetylation) were both supplied by Aldrich. Aniline (Aldrich) was distilled under reduced pressure prior to use. Ammonium persulfate (APS), hydrochloric acid ( $\mathrm{HCl}, 37 \mathrm{wt} . \%)$, acetic acid (AcA), sodium hydroxide $(\mathrm{NaOH})$, potassium bromide $(\mathrm{KBr})$ were all analytical grade reagents (Aldrich) and used as received.

\subsection{Synthesis of PANI nanoparticles}

When PANI was produced in the presence of PVP or PVA ( $4 \mathrm{wt} . \%$ ), the aqueous solution of each stabilizing polymer was prepared the day before, by stirring the mixture at controlled temperature, 40 and $80^{\circ} \mathrm{C}$, respectively. In a typical preparation $100 \mathrm{ml}$ of the aqueous solution of PVP (or PVA) and $5 \mathrm{ml}$ of $\mathrm{HCl}$ solution were added and the mixture was thoroughly deoxygenated under a constant flux of gaseous nitrogen. Afterwards, $1 \mathrm{ml}$ of aniline was added and the reactor was kept refrigerated at $4{ }^{\circ} \mathrm{C}$, while continuously stirring under a nitrogen flux. The polymerization was initiated by the slow addition of $50 \mathrm{ml}$ of APS ( $5.3 \mathrm{~g}$ ) aqueous solution. The reaction was carried out for $8 \mathrm{~h}$. After synthesis, the obtained dispersions were kept sealed within their reactors and stored at $4{ }^{\circ} \mathrm{C}$ under nitrogen until they were used for characterization. The two colloidal dispersions are referred as "PVP-PANI" and "PVA-PANI" and the corresponding $\mathrm{pHs}$ are 1.5 and 1.7, respectively.

Polyaniline was also synthesized in the same experimental conditions as above, but in the absence of stabilizing polymers for comparison and named after "bulk PANI".

When aqueous chitosan (2 wt.\%) was used as reaction medium, the solution $(100 \mathrm{ml})$ was prepared at room temperature and in the presence of acetic acid (CT/AcA $0.1 \mathrm{wt} . \%, \mathrm{pH} \sim 4$ ). Aggregates have been removed by means of filtration through $5,1.2,0.8$ and $0.45 \mu \mathrm{m}$ pore size membranes, successively. This solution was then added of aqueous $\mathrm{HCl}(\mathrm{pH}=0.2)$, aniline and APS, as for the other two preparations. The corresponding system is named after "CTPANI". Syntheses were carried out in triplicate and conversion of aniline into PANI was assessed both gravimetrically and by means of HPLC analysis of the sol fraction. Conversion resulted always very high (92-96\%) and residual aniline content minimal.

In order to remove any eventual oligomers formed, nanoparticle dispersions were dialyzed against $\mathrm{HCl}$-water $(1 \mathrm{M})$ with $12 \mathrm{k}$ MWCO cellulose dialysis tubes (Sigma Aldrich). In order to convert PANI from emeraldine salt to emeraldine base $(\mathrm{pH}>4)$, a portion of the dialyzed dispersions were first neutralized with aqueous sodium hydroxide and then dialyzed against distilled water (CTPANI) or aqueous sodium hydroxide at pH 9 (PVP-PANI and PVA-PANI). All the stabilizing polymers have higher molecular weight than the dialysis membrane cut-off, therefore surfactants have not been removed during dialysis, as also confirmed by gravimetric measurements on the solid prior and after each dialysis step. Volume variations of dispersions upon dialysis can also be considered negligible.

\subsection{Characterizations}

FTIR analysis was carried out with Perkin Elmer-Spectrum 400 apparatus by mixing with potassium bromide and then air-drying a given volume of the aqueous dispersion after dialysis and compressing it into pellets. Spectra were recorded at 32 scans per spectrum at $1 \mathrm{~cm}^{-1}$ resolution in the $4000-400 \mathrm{~cm}^{-1}$ range. The weight ratio between the suspending polymer and PANI is approximately 4 for the PVP-PANI and PVA-PANI systems and 2 for CT-PANI.

TGA was performed with a STA6000 Simultaneous Thermal Analyzer, PerkinElmer, in gaseous nitrogen flux on approx. 15-20 mg samples of the polymeric stabilizers and the corresponding colloidal PANI air-dried systems, as obtained after dialysis. A temperature ramp from $30^{\circ} \mathrm{C}$ to $950{ }^{\circ} \mathrm{C}$ was programmed with a heating rate of $10^{\circ} \mathrm{C} / \mathrm{min}$. For chitosan and PANI-chitosan systems, in consideration of the high solid residues after the ramp, the measurement was also performed at $5{ }^{\circ} \mathrm{C} / \mathrm{min}$ with a final dwell period of $10 \mathrm{~min}$ at $950^{\circ} \mathrm{C}$.

DLS measurements were carried out on PANI aqueous dispersions to obtain information on the particle size on the PANI 
nanocolloids as function of $\mathrm{pH}$. Samples were put into a thermostated cell compartment of a Brookhaven Instruments BI200-SM goniometer. The temperature was controlled to within $0.1^{\circ} \mathrm{C}$ using a thermostated recirculation bath. The light scattered intensity and time autocorrelation function were measured by using a Brookhaven BI-9000 correlator and a $100 \mathrm{~mW}$ Ar laser (Melles Griot) tuned at $\lambda=632,8 \mathrm{~nm}$. Measurements were taken at different scattering vector $q=4 \pi n \lambda / \sin (\theta / 2)$, where $n$ is the refraction index of the solution, $\lambda$ is the wavelength of the incident light, and $\theta$ is the scattering angle. Dynamic light scattering data were analyzed by the method of cumulants. Mean hydrodynamic radius of nanoparticles and standard deviation have been calculated accordingly [23].

Transmission electron microscopy (TEM) was carried out with a JEOL-2100 at $80 \mathrm{kV}$ of accelerating voltage on dispersion samples dropped onto holey carbon-coated copper grids. In order to resolve the morphology of the individual particles in the dry form, thus avoiding uncontrolled PANI particles agglomeration, the content of the suspending polymer in dispersion could not be lowered indiscriminately. Therefore, dispersions were observed (i) after a ten-fold dilution with the corresponding stabilizing polymer aqueous solution (solid content: $4 \mathrm{wt} . \%$ and $2 \mathrm{wt} . \%$; surfactant/PANI weight ratio 40 and 20 for PVA-PANI (PVP-PANI) and CT-PANI, respectively); (ii) after 500 times dilution using a tenfold diluted stabilizing polymer solutions (solid content: $0.4 \mathrm{wt} . \%$ and 0.2 wt.\%; surfactant/PANI weight ratio 200 and 100 for PVA-PANI (PVP-PANI) and CT-PANI respectively); and (iii) after dilution as in (i) and (ii) but using stabilizing polymer solutions whose $\mathrm{pH}$ was previously modified by addition of concentrated aqueous $\mathrm{NaOH}(6 \mathrm{M})$ or $\mathrm{HCl}$. Generally, the addition of $\mathrm{NaOH}$ or $\mathrm{HCl}$ has been followed by dialysis with alkaline or acid aqueous solution at the target $\mathrm{pH}$ for desalting.

Conductivity measurements were carried out via Impedance Spectroscopy (IS) by means of a Frequency Response Analyser (FRA, Schlumberger, mod.1255), at room temperature, in the frequency range $10^{-1}-10^{5} \mathrm{~Hz}$, applying a $0.02 \mathrm{~V}$ peak-to-peak ac signal. A two electrode cell with two circular $20.5 \mathrm{~mm}$ gold plated electrodes (working and counter) was used. Samples in the form of circular pastilles of $21 \mathrm{~mm}$ diameter and $\sim 1 \mathrm{~mm}$ thickness were obtained by compression molding with a manual hydraulic press the air-dried solids, as obtained from the dialyzed dispersions at $\mathrm{pH}$ 1. Pastilles were gently pressed between the electrodes in order to ensure the electrical contact. Measuring cell and apparatus were placed into a Faraday cage for shielding electromagnetic interference.

UV-Vis absorption spectra were carried out by using a UV-2401 PC Shimadzu Spectrophotometer at room temperature, scan speed of $40 \mathrm{~nm} / \mathrm{min}$, integration time $2 \mathrm{~s}$ and bandwidth $1 \mathrm{~nm}$. Original dispersions were diluted 10 times and 1000 times using a tenfold diluted stabilizing polymer solution whose $\mathrm{pH}$ was modified by addition of concentrated $\mathrm{NaOH}(6 \mathrm{M})$.

Fluorescence measurements were carried out with a JASCO FP6500 spectrofluorimeter equipped with a Xenon lamp (150 W).

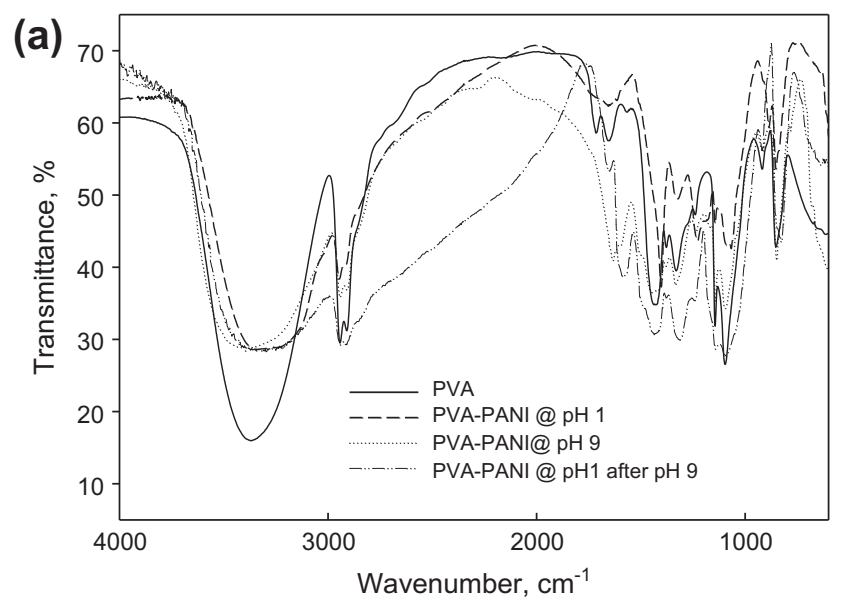

(c)
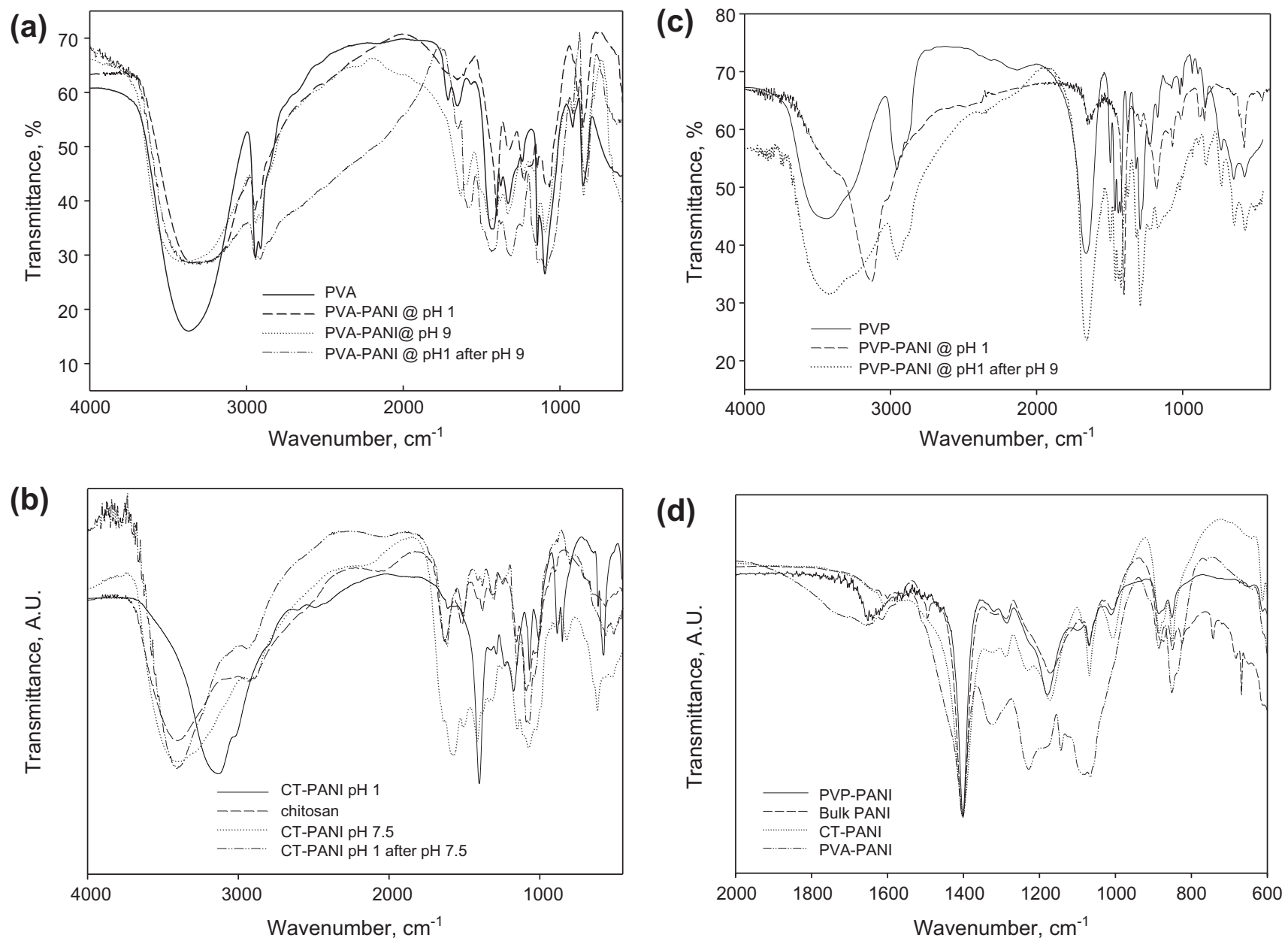

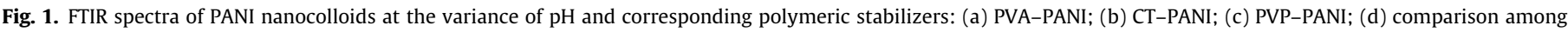
spectra of different colloidal PANIs and bulk PANI, i.e. polyaniline synthesized in the absence of polymeric stabilizer. 
Table 1

Wavenumbers of various vibration peaks observed and corresponding assignment of vibrations for pure PVP and PVP-PANI nanocolloids.

\begin{tabular}{|c|c|c|c|}
\hline System & $\begin{array}{l}\text { Wavenumber } \\
\left(\mathrm{cm}^{-1}\right)\end{array}$ & Assignment & Remarks \\
\hline \multirow[t]{5}{*}{ PVP } & $3700-3000$ & $v \mathrm{O}-\mathrm{H}$ and $v \mathrm{~N}-\mathrm{H}$ & $\begin{array}{l}\text { Multiband envelope due to stretching vibration modes of hydroxyl and amidic groups and their } \\
\text { association stemming from hydration of pyrrolidone groups }\end{array}$ \\
\hline & $\begin{array}{l}2956 ; 2925 ; \\
2888\end{array}$ & $v \mathrm{C}-\mathrm{H}$ & $\begin{array}{l}\text { Stretching bands of methyl and methylene groups of both polymeric backbone and pyrrolidone } \\
\text { rings }\end{array}$ \\
\hline & 1663 & $v C=0$ & $\begin{array}{l}\text { Tertiary amide generally positioned at } 1650 \mathrm{~cm}^{-1} \text {, here shifted of ca. }+10 \mathrm{~cm}^{-1} \text { due to water } \\
\text { mediated association }\end{array}$ \\
\hline & $\begin{array}{l}1498 ; 1465 ; \\
1443 ; 1378\end{array}$ & $\begin{array}{l}\delta_{\mathrm{s}}-\mathrm{CH}_{2}-; \delta_{\mathrm{as}}-\mathrm{CH}_{3} \text { and } \\
\delta_{\mathrm{s}}-\mathrm{CH}_{3} ;-\mathrm{CH}_{2}-(\mathrm{C}=\mathrm{O}) \\
\text { and }-\mathrm{CH}_{2}-\mathrm{N}^{+}\end{array}$ & $\begin{array}{l}\text { Bending bands of methylene and methyl groups, also in proximity of electron withdrawing } \\
\text { groups }\end{array}$ \\
\hline & $\begin{array}{l}1321 ; 1293 ; \\
1257 ; 1204\end{array}$ & $v C-N$ & Stretching bands of $\mathrm{C}-\mathrm{N}$ in pyrrolidone rings \\
\hline \multirow[t]{6}{*}{$\begin{array}{l}\text { PVP-PANI } \\
\text { (Emeraldine-salt) }\end{array}$} & $3600-2600$ & $\begin{array}{l}v \mathrm{O}-\mathrm{H}, v \mathrm{~N}-\mathrm{H} \text { and } \\
\mathrm{N}^{+}-\mathrm{H}, \mathrm{vC}-\mathrm{H}\end{array}$ & $\begin{array}{l}\text { Multiple contributions coming from PVP (hydroxyl groups of bound water, methyl and methylene } \\
\text { stretching vibrations) and PANI. Pronounced new peaks at } 3143 \text { and shoulder at } 3050 \mathrm{~cm}^{-1} \text { due to } \\
\phi-\mathrm{NH}-\phi \text { or } \phi-\mathrm{NH}-\mathrm{R} \text { and } \phi-\mathrm{H} \text {, respectively. The absorptions characteristic of hydration in PVP is } \\
\text { less evident, probably due to the increased hydrophobicity of the composite system coming from } \\
\text { PANI }\end{array}$ \\
\hline & 1652 ; & $v \mathrm{C}=\mathrm{O}$ & $\begin{array}{l}\text { PVP carbonyl group stretching mode, shifted down to lower wavenumbers, for the reduced } \\
\text { interaction of the carbonyl group with water molecules }\end{array}$ \\
\hline & 1403 & $v \mathrm{NH}_{4}^{+}$ & $\begin{array}{l}\text { Very strong band. When in association with absorptions in the region } 3100-3500 \mathrm{~cm}^{-1} \text { it can be } \\
\text { attributed to the stretching vibration of ammonium ions, that may derive from ammonium } \\
\text { sulfate }\end{array}$ \\
\hline & $\begin{array}{l}1333-1291 ; \\
1181\end{array}$ & $v_{a s} C-N$ and $v_{s} C-N$ & $\begin{array}{l}\text { Several bands attributable to aromatic and aliphatic amines, that split into more components for } \\
\text { the coexistence of structures differing by conformation and/or charge configuration (e.g. } \mathrm{C}-\mathrm{NH}^{+} \text {; } \\
\mathrm{C}=\mathrm{NH}^{+} \text {). In particular, absorption at about } 1300 \mathrm{~cm}^{-1} \text { corresponds to } \pi \text {-delocalization induced by } \\
\text { protonation }\end{array}$ \\
\hline & $\begin{array}{l}1133-1084, \\
888\end{array}$ & $\delta_{\text {(in-plane) }} \mathrm{C}-\mathrm{H}$ & In plane and out-of-plane bending of $\mathrm{C}-\mathrm{H}$ \\
\hline & & $\delta_{\text {(out-of-plane) }} \mathrm{C}-\mathrm{H}$ & \\
\hline
\end{tabular}

Table 2

Wavenumbers of various vibration peaks observed and corresponding assignment of vibrations for pure PVA and PVA-PANI nanocolloids.

\begin{tabular}{|c|c|c|c|}
\hline System & Wavenumber $\left(\mathrm{cm}^{-1}\right)$ & Assignment & Remarks \\
\hline \multirow[t]{4}{*}{ PVA } & $\sim 3400$ & $\mathrm{vO}-\mathrm{H}$ & Broad band due to polymeric hydroxyl groups, also hydrogen bonded \\
\hline & $2948 ; 2938$ & $v C-\mathrm{H}$ & Methylene groups \\
\hline & $1725 ; 1670$ & $v C=0$ & Acetate groups \\
\hline & $1450 ; 1341 ; 1146 ; 1103$ & $\delta \mathrm{C}-\mathrm{H} ; \mathrm{vC}-\mathrm{O}$ & Characteristic absorptions of alkane groups and alcohols \\
\hline \multirow[t]{6}{*}{ PVA-PANI (Emeraldine salt) } & $3700-2600$ & $v \mathrm{O}-\mathrm{H}, v \mathrm{C}-\mathrm{H} v \mathrm{~N}-\mathrm{H}$ and $v \mathrm{~N}^{+}-\mathrm{H}$ & $\begin{array}{l}\text { Multi-component absorption band including polymeric hydroxyl groups, } \\
\mathrm{N}-\mathrm{H}, \mathrm{C}-\mathrm{H} \text { and } \phi-\mathrm{H} \text { stretching modes of PVA and PANI }\end{array}$ \\
\hline & $1727 ; 1677$ & $v C=0$ & As in pure PVA \\
\hline & 1406 & $v \mathrm{NH}_{4}^{+}$ & From PANI, as in PANI-PVP \\
\hline & $1340 ; 1237-1200$ & $v C-N$ & From PANI, as in PANI-PVP \\
\hline & $1146 ; 1099-1089 ; 890$ & $\delta_{\text {(in-plane) }} \mathrm{C}-\mathrm{H}$ & From PANI, as in PANI-PVP \\
\hline & & $\delta_{\text {(out-of-plane) }} \mathrm{C}-\mathrm{H}$ & \\
\hline
\end{tabular}

Table 3

Wavenumbers of various vibration peaks observed and corresponding assignment of vibrations for pure chitosan and CT-PANI nanocolloids.

\begin{tabular}{|c|c|c|c|}
\hline System & Wavenumber $\left(\mathrm{cm}^{-1}\right)$ & Assignment & Remarks \\
\hline \multirow[t]{6}{*}{ Chitosan } & $3700-3100$ & $v \mathrm{O}-\mathrm{H}, \mathrm{vN}-\mathrm{H}$ & $\begin{array}{l}\text { Contributions from polymeric and hydrogen-bonded } \\
\text { hydroxyl groups and amino groups }\end{array}$ \\
\hline & $2965 ; 2918$ & $v \mathrm{C}-\mathrm{H}$ & Aliphatic groups \\
\hline & 1643 & $v C=0$ & Amide I band of GlcNAc units \\
\hline & 1529 & $\delta \mathrm{N}-\mathrm{H}$ & $\begin{array}{l}\mathrm{R}-\mathrm{NH}_{2} \text { of } \mathrm{GlcN} \text { units, shifted to lower wavenumbers due } \\
\text { to hydrogen bonding and/or association }\end{array}$ \\
\hline & $1430 ; 1391$ & $\delta \mathrm{C}-\mathrm{H}$ & $\begin{array}{l}\text { Ordinary bending bands of methylene and methyl } \\
\text { groups }\end{array}$ \\
\hline & $1335 ; 1267 ; 1160$ & $v_{\mathrm{asc}-\mathrm{N}}$ and $v_{\mathrm{sC}-\mathrm{N}}$ & Aliphatic amines \\
\hline \multirow[t]{3}{*}{ CT-PANI (Emeraldine salt) } & $3150-3053$ & $v \mathrm{O}-\mathrm{H}, \mathrm{vN}-\mathrm{H}$ & Prevalence of absorption bands from PANI \\
\hline & 1405 & $v \mathrm{NH}_{4}^{+}$ & From PANI, as in PANI-PVP \\
\hline & $\begin{array}{l}1335 ; 1295 ; 1240-1180 \\
1070 ; 887-879\end{array}$ & $\begin{array}{l}v_{\text {asC }-\mathrm{N}} \text { and } v_{\mathrm{sC}-\mathrm{N}} v_{\text {as } \mathrm{c}-\mathrm{O}-\mathrm{c}} \text { and } v_{\mathrm{s} \mathrm{c}-\mathrm{O}-\mathrm{c}} \\
\delta_{\text {(in-plane) }} \mathrm{C}-\mathrm{H} \\
\delta_{\text {(out-of-plane) } \mathrm{C}-\mathrm{H}}\end{array}$ & $\begin{array}{l}\text { Present both in chitosan and PANI } \\
\text { From PANI, as in PANI-PVP }\end{array}$ \\
\hline
\end{tabular}

Emission and photoluminescence excitation (PLE) spectra, at the required excitation, were obtained with emission and excitation bandwidths of $3 \mathrm{~nm}$, scan-speed of $100 \mathrm{~nm} / \mathrm{min}$ and integration time of $1 \mathrm{~s}$, recorded at $0.5 \mathrm{~nm}$ intervals. The experimental errors were about $2 \%$. Measurements were carried out on 1000 times diluted dispersions at the variance of $\mathrm{pH}$ as well as on the 
corresponding suspending polymer solutions. Nanocolloids showed an emission band at $320 \mathrm{~nm}$, when excited at $290 \mathrm{~nm}$. Absolute values of the emission intensity at peak were $\sim 300$ at $\mathrm{pH} 1$ and $\sim 170$ for pHs between 2.4 and 5, for PVA-PANI; negligible emissions were observed at pHs above 6. CT-PANI nanocolloids' emissions were always $\sim 160$ for all pHs up to 6 , when precipitation occurs. Emission spectra were normalized for the absorbance values at the corresponding excitation wavelength.

\section{Results and discussion}

\subsection{Structural and thermal analyses}

Fig. 1a-c shows FTIR spectra of PVP-PANI, PVA-PANI and CTPANI nanocolloids after dialysis at $\mathrm{pH} \mathrm{1,} \mathrm{pH} 9$ and back to $\mathrm{pH} 1$. The spectra of the corresponding stabilizing polymers are also reported for comparison. Fig. 1d presents a direct comparison between the three colloidal PANI and the "bulk PANI", synthesized in the same conditions but in the absence of stabilizer, at $\mathrm{pH} \sim 1$. Attribution of the main absorbance bands of the stabilizers and of the solid residues of PANI NPs nanocolloids is reported in Tables 1-3.

All PANI containing systems (Fig. 1a-c) show common spectroscopic features, that are attributable to the presence of PANI in the form of emeraldine salt [24-28] and retain some other structural features that are specific of the stabilizing polymer used. A broad and intense peak at $3140 \mathrm{~cm}^{-1}$ with a shoulder at $3050 \mathrm{~cm}^{-1}$, a prominent absorption at about $1400 \mathrm{~cm}^{-1}$, bands at $1330-1320$ and $1285 \mathrm{~cm}^{-1}, \quad \sim 1180 \mathrm{~cm}^{-1}, \quad 1090-1070 \mathrm{~cm}^{-1}$ and 890 $850 \mathrm{~cm}^{-1}$ are displayed by all colloidal PANIs produced. These bands are also present in the spectrum of PANI produced in the absence of stabilizer (Bulk-PANI) and at the same APS/aniline ratio, aniline concentration, $\mathrm{pH}$ and temperature, as shown in Fig. 1d. Conversely, in the $650-480 \mathrm{~cm}^{-1}$ region, all colloidal PANI show sharper and well resolved bands, as it is often observed in nanoscopic systems [27], differently from to the bulky polymer.

In particular, absorptions in the high wavenumber range $(3140$ and $3050 \mathrm{~cm}^{-1}$ ) of PANI containing systems may be attributed to the presence of benzene ring adjacent to amine groups and to protonated amines $\left(--\mathrm{NH}-\mathrm{C}_{6} \mathrm{H}_{4}-\mathrm{NH}-\right.$ and $\left.-\mathrm{NH}_{2}^{+}-\mathrm{C}_{6} \mathrm{H}_{4}-\mathrm{NH}_{2}^{+}-\right)$. These broad absorptions are accompanied by hydroxyl groups stretching vibrations enlarging the $\mathrm{N}-\mathrm{H}$ absorption envelop toward the higher wavenumbers, owing to residual hydration water and additionally to polymeric hydroxyl groups, for PVA-PANI only.

Quite interestingly, typical absorptions of ring modes near 1600 and $1500 \mathrm{~cm}^{-1}$ (quinonoid and benzenoid ring-stretching deformations of PANI) [24] are not evident in any of the spectra of PANI as produced, when $\mathrm{pH}$ has been maintained acid from synthesis to purification. The most intense vibration band stemming from the presence of PANI is the peak at $\sim 1400 \mathrm{~cm}^{-1}$ (see Fig. 1d).

Attribution of this peak is not straightforward: it can be associated to protonated nitrogen atoms of the polymer backbone or to ammonium sulfate by-product, deriving from APS, and incorporated as "ionic crosslinker" among PANI chains and/or among PANI and the polymeric stabilizer chains. Strong, non-covalent, interaction between polyaniline and polymeric surfactant, either direct or sulfate ions mediated, may also be responsible for the little spectroscopic evidence of the characteristic absorption bands of the functional groups of the stabilizer in the nanocolloids at synthesis $\mathrm{pH}$, although the polymeric stabilizers are present as the major component. Their appearance upon basification at $\mathrm{pH} 9$ and clear evidence, although shifted, also at $\mathrm{pH} 1$ upon re-acidification, support the non-covalent attachment of PANI. In particular, for PVA-PANI dispersions (Fig. $1 \mathrm{a}$ ) at $\mathrm{pH}=9$ the sharp band peaking at about $1400 \mathrm{~cm}^{-1}$ disappears and two new bands appear: at $1451 \mathrm{~cm}^{-1}$ with a shoulder at $1490 \mathrm{~cm}^{-1}$, and at $1635 \mathrm{~cm}^{-1}$ with a shoulder at $1580 \mathrm{~cm}^{-1}$. The two shoulders are attributable to benzenoid and quinonoid groups of PANI, respectively, while the main bands are attributable to PVA. When $\mathrm{pH} 1$ is brought back to acid, the strong absorption at $1400 \mathrm{~cm}^{-1}$ is not restored, therefore it is very likely that the dialysis at $\mathrm{pH} 9$, reducing the positive charge density of PANI chains, allows ammonium sulfate ions to be displaced off upon dialysis. Interestingly, in this case the high wavenumber portion of the spectrum presents a broad band between $2800 \mathrm{~cm}^{-1}$ and $2000 \mathrm{~cm}^{-1}$, characteristic of conjugation. Similar results have been observed for CT-PANI and PVP-PANI (Fig. 2b and c). Therefore, it can be concluded that ammonium sulfate is responsible for most of the signal at $1400 \mathrm{~cm}^{-1}$ in the spectra of all colloidal systems at their "original" low $\mathrm{pH}$. When emeraldine salt is converted into its base form, the characteristic
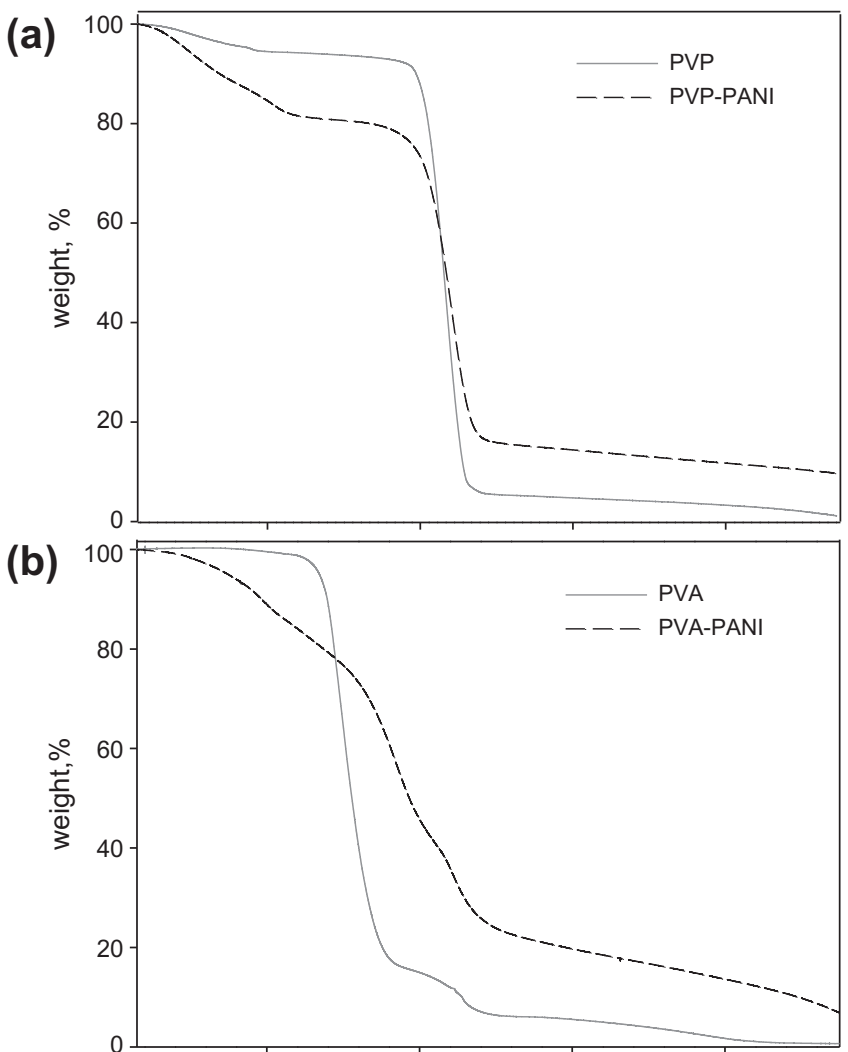

(c)

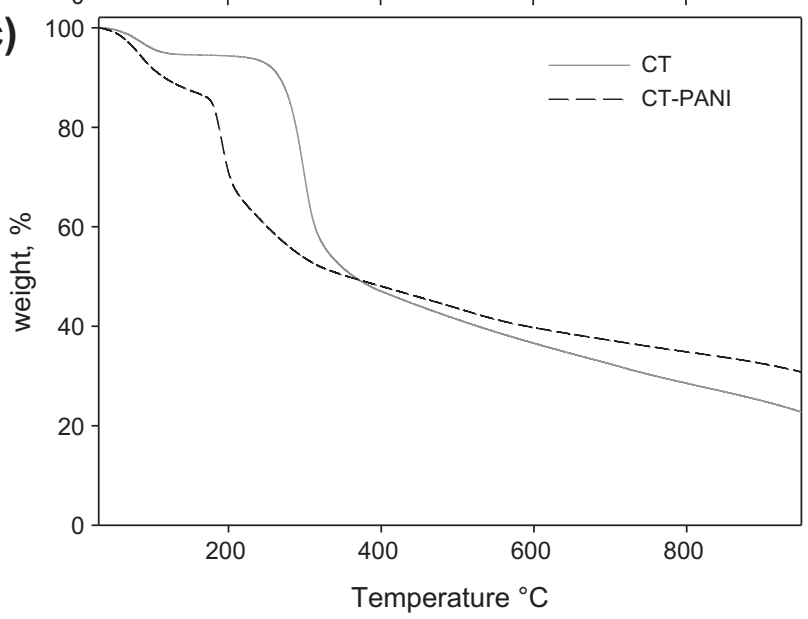

Fig. 2. TGA curves of air-dried PANI nanocolloids and corresponding polymeric stabilizers under nitrogen (heating rate $10^{\circ} \mathrm{C} / \mathrm{min}$ ): (a) PVP and PVP-PANI; (b) PVA and PVA-PANI; (c) CT and CT-PANI. PANI is in the form of protonated emeraldine. 

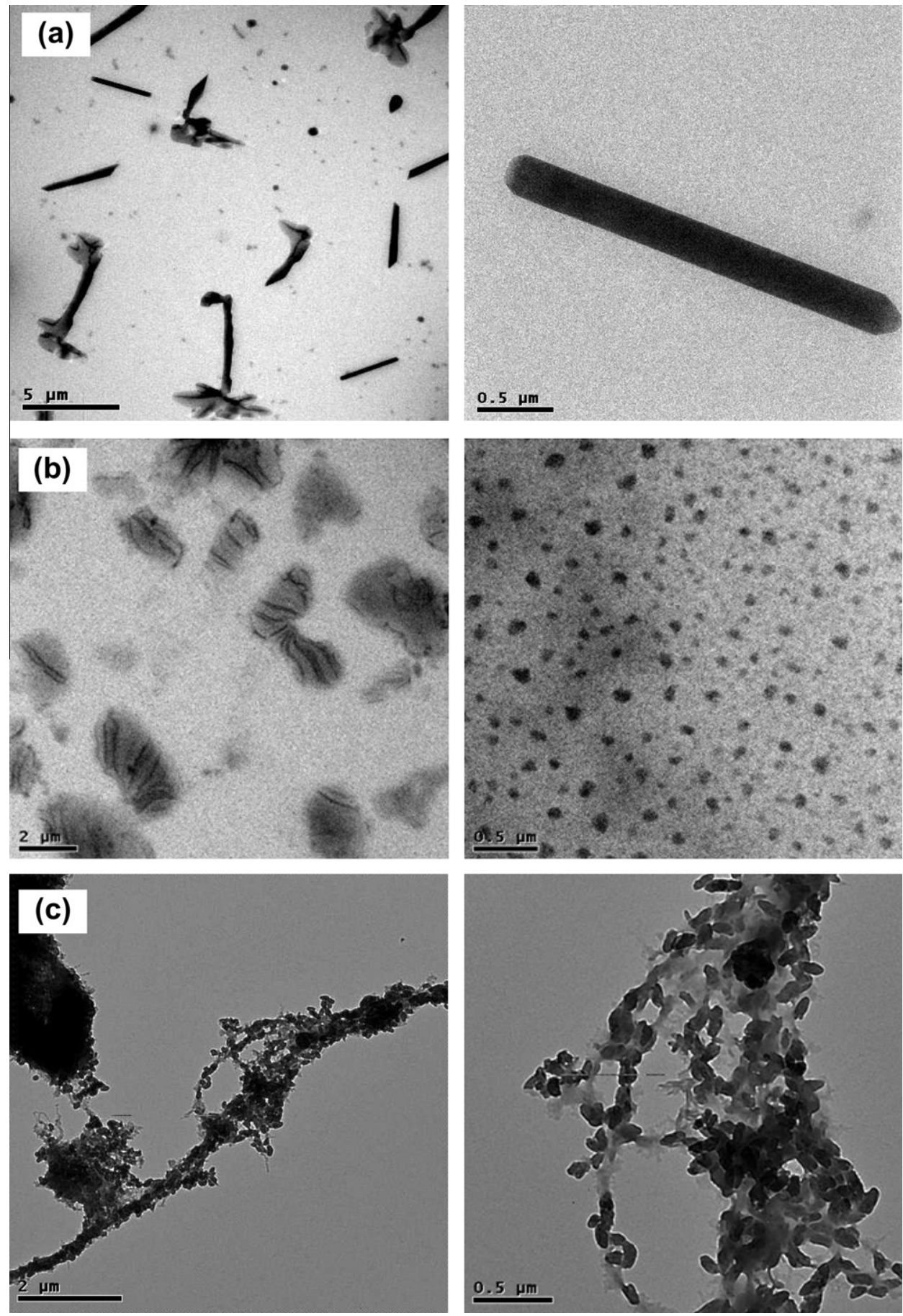

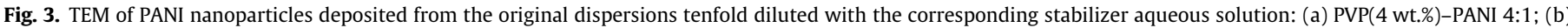
PVA(4 wt.\%)-PANI 4:1; (c) CT(2 wt.\%)-PANI 2:1.

absorption bands of the stabilizer polymer in the nanocomposite become much more evident, as well as bands that are characteristic of benzenoid and quinonoid rings of PANI. This modification appears to be irreversible, as the pristine spectra are never restored after re-acidification.

Fig. 2a-c shows the thermo-gravimetric curves of the different PANI nanocolloids and the corresponding stabilizing polymers for reference. It is worth pointing out that the stabilizers are the major constituents of the nanocomposites, but the in situ aniline polymerization process and the presence of the formed polyaniline in the dry solids can be both responsible for the evident modifications of the TGA curves when compared to those of the pure polymers. More pronounced initial weight decrements are shown by the nanocolloids for the higher amounts of residual moisture and bound water, owing to the ionic character induced by PANI in the form of emeraldine salt. Then, the loss of hydrogen chloride, present as doping agent, may contribute to the further weight decrements up to about $350^{\circ} \mathrm{C}$ [25], as well as the thermal degradation of any eventual lower molecular weight tails of the stabilizing polymers (higher than $12 \mathrm{k}$, that is the dialysis membranes cut-off) produced by oxidative degradation upon aniline polymerization and eventual adducts formed with oligomeric 

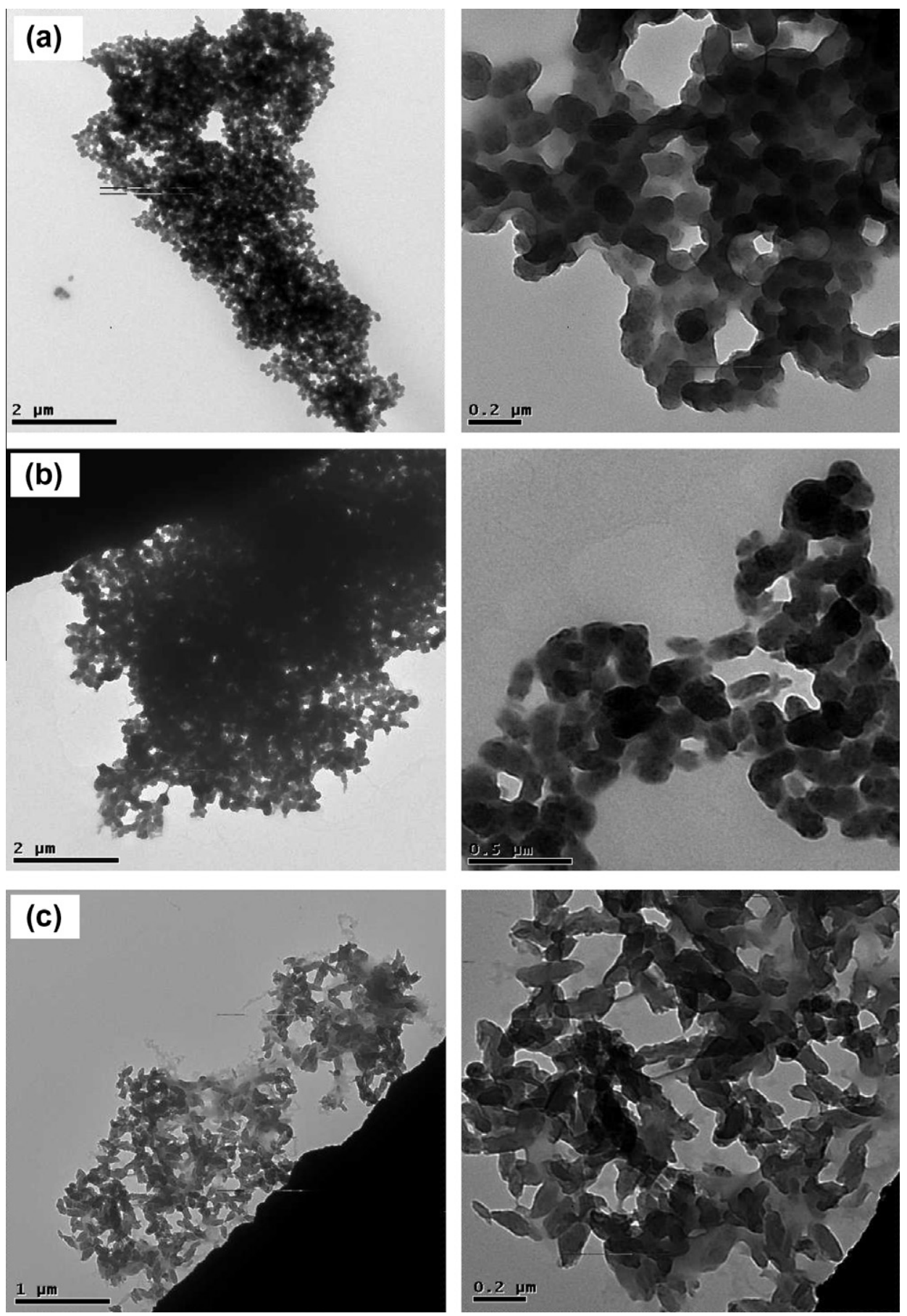

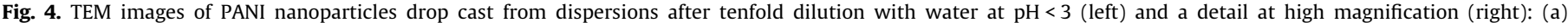
PVP(0.4 wt.\%)-PANI/40:1; (b) PVA(0.4 wt.\%)-PANI/40:1; (c) CT(0.2 wt.\%)-PANI/20:1.

PANI. This contribution is more important for the natural sourced chitosan, with respect to synthetic PVP or PVA. In order to rule out any artifact from measurement conditions, TGA curves for CT and CT-PANI have been also produced at a lower heating rate $\left(5{ }^{\circ} \mathrm{C} / \mathrm{min}\right)$, the only appreciable difference being the expected shift toward the lower temperatures of the characteristic onset and offset of the observed phenomena (data not shown). Evolution of acid vapors may accelerate degradation of the polymeric surfactants too as they do for PANI itself [25]. Degradation of macromolecular PANI, generally occurring between 350 and $450{ }^{\circ} \mathrm{C}$, is here super- imposed with the main degradation of the suspending polymers. In this interval, all nanocolloids' curves show a crossover with those of the stabilizers, thus suggesting that PANI nanoparticles are contributing to increase the thermal stability of the stabilizing polymers and to the final solid residues. It is interesting to note that the residual weight for PVA-PANI is approx. $8 \mathrm{wt} . \%$, while pure PVA present nil, and this value is always equal to the difference of the residuals of the two other nanocolloids and their corresponding stabilizers, thus suggesting approximately the same amount of PANI in all systems. 


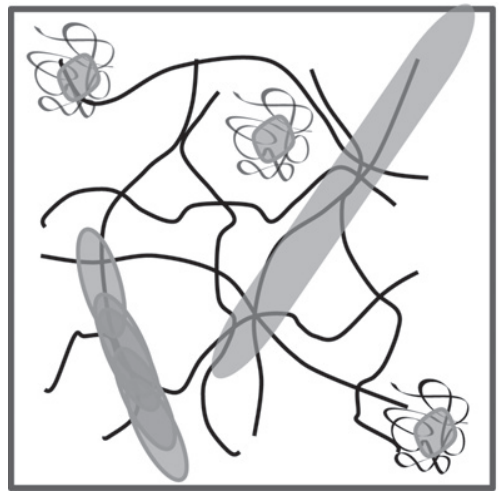

(a)

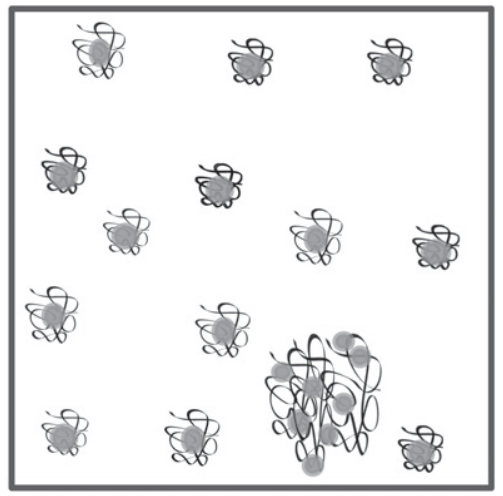

(b)

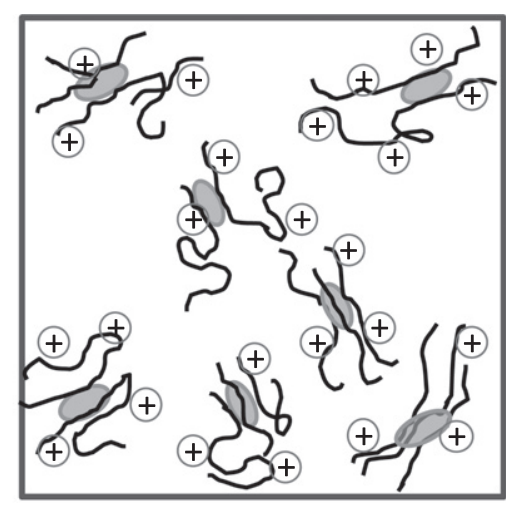

(c)

Scheme 1. (a) PVP-PANI; (b) PVA-PANI; (c) CT-PANI.

\subsection{Morphological analysis}

\subsubsection{Influence of the nature of the stabilizing polymer}

The morphology of colloidal PANI has been investigated via DLS and TEM. It is widely recognized that water-soluble polymers may effectively provide steric stability for the growth of polymer nanoparticles during chemical oxidative polymerization of aniline [29]. In the early stages of polymerization "primary particles" are formed, whose size and molecular structure is dictated by the synthetic conditions, i.e. reaction feed composition, $\mathrm{pH}$, temperature, nature of the oxidizing molecule and doping agents [26]. Their evolution toward more complex forms strongly depends on the nature and extent of interactions with the stabilizing polymer. When aniline is polymerized in the presence of a water-soluble stabilizer, then the precipitating chains get attached (either grafted or adsorbed) to the steric stabilizer. These "primary particles" further grow outwardly through aggregation, with the steric stabilizer being incorporated inside them [30]. This aspect is key for the control of the production process and final properties of the polymer nanoparticles obtained [31-33]. Fundamental understanding of how particle size and morphological changes influence the physico-chemical properties is still a matter of high technological interest [34].

PANI nanoparticles mean hydrodynamic diameters and standard deviations have been determined by DLS as function of $\mathrm{pH}$ and at the variance of the scattering angle. It is worth pointing out that the estimated hydrodynamic diameter refers to PANI particles with their polymeric surfactant, hydrated, suspending shell.

For PVP-PANI, the calculated average hydrodynamic diameters $\left(D_{h}\right)$ at $90^{\circ}$ and $\mathrm{pH} 1,7.5$ and 9 were $430 \pm 95 \mathrm{~nm}, 500 \pm 125 \mathrm{~nm}$ and $440 \pm 90 \mathrm{~nm}$, respectively. Similar data were obtained for measurements at $40^{\circ}$. At $150^{\circ} D_{h}$ were $300 \pm 100 \mathrm{~nm}, 600 \pm 100 \mathrm{~nm}$ and $330 \pm 60 \mathrm{~nm}$ for the three $\mathrm{pH}$ in the same order. The observed differences in $D_{h}$ at the variance of the scattering angle suggest a high degree of size polydispersity at all pHs. For PVA-PANI, $D_{h}$ resulted to be $300 \pm 70 \mathrm{~nm}, 340 \pm 70 \mathrm{~nm}$ and $520 \pm 50 \mathrm{~nm}$ for $\mathrm{pH} \mathrm{1,} 7.5$ and 9 , respectively. Small quantities of a precipitate is observed especially in alkaline conditions. For CT-PANI dispersion analyzed in the same conditions, $D_{h}$ resulted $300 \pm 70 \mathrm{~nm}$ at $\mathrm{pH} 1$, while the dispersed solid fully precipitates at $\mathrm{pH}$ 7.5.

The $D_{h}$ values measured for both PVA-PANI and CT-PANI were almost invariant with the scattering angle, suggesting a better control of PANI particle size.

TEM analysis of the dry deposit show for PVP(4 wt.\%)-PANI, Fig. 3a, a predominant number of micron-long rods, as well as sub-micrometric, quasi-spherical particles and some rods with branched arms at their extremities, that appear to result from Ostwald ripening-like phenomena. This picture suggests a role for the PVP as "soft template" in the growth of polyaniline chains and/or PANI particles supramolecular organization.

In facts, from dynamic light scattering experiments carried out on the aqueous solutions of the same PVP [35], it was established that at a concentration of $4 \mathrm{wt} . \%, \mathrm{PVP}$ is in the semi-dilute regime and above the chain overlapping concentration $\left(C^{*}=1 \mathrm{wt} . \%\right)$. PVP aqueous solution can be described as formed by freely moving coils, that may correspond to single PVP chains or small aggregates of few chains, and a transient polymer network, i.e. a network formed by labile junctions due to van der Waals interactions, water-mediated intermolecular hydrogen bonds and/or chain entanglements. Stabilization of PANI particles operated by PVP occurs mainly through the establishment of hydrogen bonding between PANI amine $(-\mathrm{NH}-$ ) groups and non-ionic carbonyls of PVP pyrrolidone rings [36], but also through electrostatic interactions between protonated imine sites $\left(=\mathrm{N}^{+}-\right.$) of PANI-ES and negatively charged carbonyl groups of PVP, that may form by keto-enol tautomerization. The organization of PVP chains in water is then reflected in the shape and size of the polyaniline particles formed, growing spherical through the establishment of interactions with PVP coils, but also in forms of elongated structures through synchronized multiple point attachment to the PVP chain-extended network, as schematically represented in Scheme 1a [37].

When the atatic, semi-crystalline PVA is used as steric stabilizer, spherical and narrow size distributed PANI NPs with diameter of about $100 \mathrm{~nm}$ are formed (Fig. 3b). Drop casting on the substrate for TEM microscopy also reveals irregular, larger composite objects of microns in size, where PANI seams intercalated into PVA crystal lamellae. PVA concentration in the reaction feed is $4 \mathrm{wt} . \%$, like for PVP, where $C^{*}$ for PVA is also estimated of about $1 \mathrm{wt} . \%$ by intrinsic viscosity measurements $\left(C^{*}=1 /[\eta]\right.$, data not reported). PVA is not as hydrophilic as PVP and it behaves in water almost as in a $\theta$-solvent [38]. Polymer chains in random coil conformation are involved in both intra- and inter-molecular aggregation, through hydrogen bonding of hydroxyl groups. It is also reported that PVA aggregates of microns in size are formed by PVA-aniline mixtures in water $[38,39]$. Upon polymerization, PANI particles may then form also inside these aggregates and associate. The situation is schematically depicted in Scheme $1 \mathrm{~b}$. This situation is believed to be the cause of the observed inferior colloidal stability of PVA-PANI dispersion with respect to PVPPANI. In fact, a PVA-PANI cake deposits from a deep green-colored supernatant phase at the bottom of the reactor when the dispersion is stored at $4{ }^{\circ} \mathrm{C}$ for longer than $48 \mathrm{~h}$ after synthesis.

With respect to PVP and PVA, chitosan is by far the most effective polymer in controlling PANI particle size and size distribution in the chosen conditions, owing to its balance of hydrophobic GlcNAc units and hydrophilic, electrically charged GlcN sites. It 

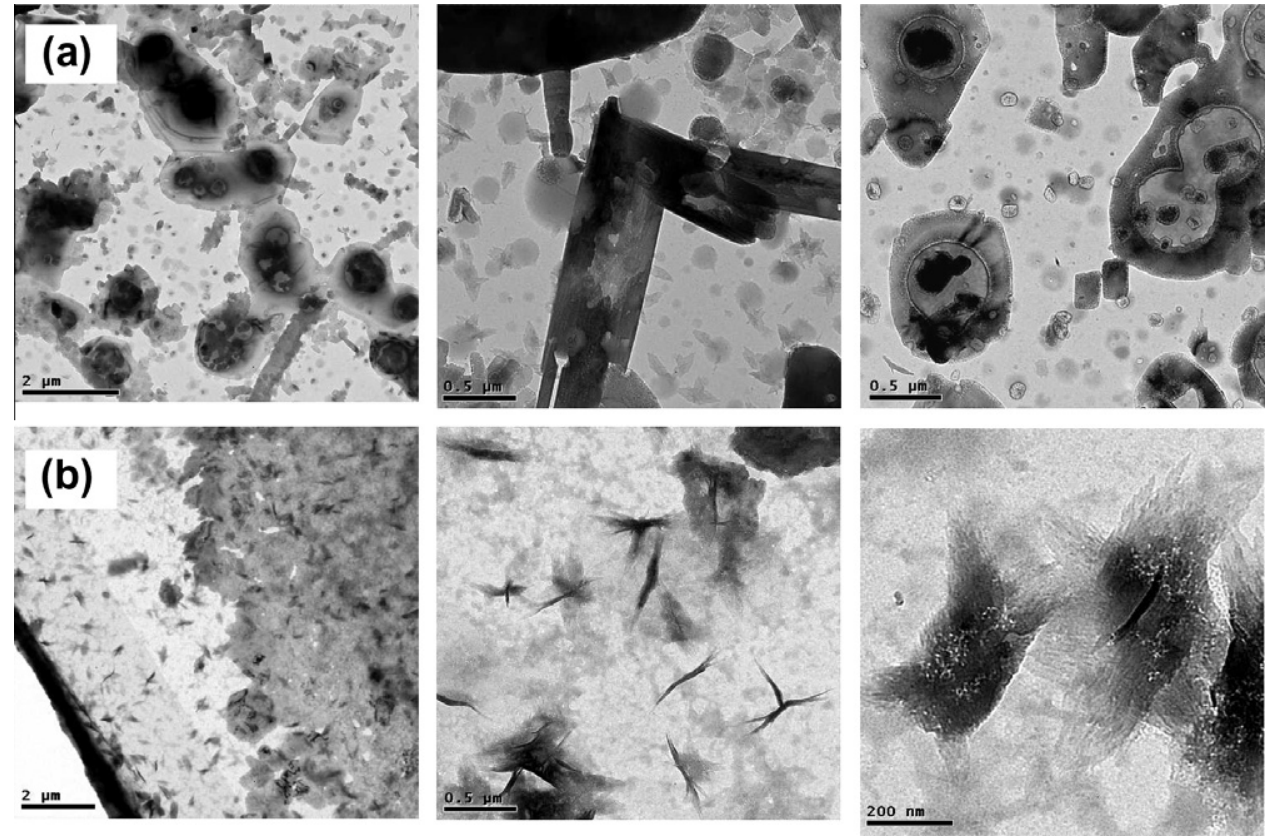

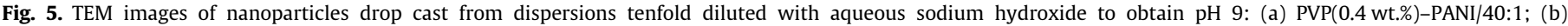
PVA(0.4 wt.\%)-PANI/40:1. The presence of structures related to $\mathrm{NaCl}$ crystals cannot be excluded.

also ensures the best dispersion stability upon storage. The polyaniline nanocomposite solution remains stable for months, that is, PANI does not fall out of the solution or forms precipitates. The drawback of the chosen variant of chitosan though is the limited water solubility and therefore the stabilizing properties are manifested only within the solubility window of chitosan $(\mathrm{pH}<6.5)$. The morphology of PANI nanoparticles obtained in the presence of chitosan is fairly uniform, Fig. 3c, with rice-grain nanoparticles of $\sim 100 \mathrm{~nm}$ for the long-axis and $\sim 10-30 \mathrm{~nm}$ for the short-axis. In consideration of the molecular characteristics of the chosen polymer (weight-average degree of polymerization, DPw $<650$; degree of acetylation $\mathrm{DA}=25-15 \%$ ), the low $\mathrm{pH}$ and the high ionic strength of the aqueous solution $(0.1 \mathrm{M} \mathrm{HCl})$, chitosan chains in solution are quite isolated at the given concentration, still presenting some degree of chain flexibility for the free chlorine ions screening of electrostatic repulsions of protonated amino-groups responsible for chain expansion [40]. Aniline oligomers should favorably absorb to the hydrophobic sites of chitosan owing to the presence of the hydrophobic GlcNAc units, that are chemically stable in the chosen reaction conditions [41], and grow uniformly (Scheme 1c).

\subsubsection{Influence of dilution and $\mathrm{pH}$}

TEM morphology of PANI nanocolloids was also investigated upon dilution with water before deposition, in order to decrease the concentration of the suspending polymer, while $\mathrm{pH}$ was always below $\sim 3$. The corresponding TEM images are reported in Fig. 4a-c. Nanoparticles with a PANI core with consistent dimensions in the range of $60-100 \mathrm{~nm}$ are present. The PVP shell cannot be distinguished. This result is quite remarkable and well in agreement with the results of DLS: the precise cylindrical structures that were observed for PANI in PVP-PANI system do not form and TEM microscopy reveal now the "primary" PANI particles [29]. Only chitosan, among the three polymeric surfactants, is effective in preserving the original morphology upon drying. Expectedly, if the same dilution is operated with aqueous sodium hydroxide to change $\mathrm{pH}$ to 9 , CT-PANI undergoes flocculation and precipitation as chitosan is no longer water-soluble.
Contrariwise, PVP-PANI and PVA-PANI dispersions at pH 9 appear macroscopically homogeneous, but TEM analysis (Fig. 5a and b) reveals the occurrence coarse coalesce and the on-set of phase separation phenomena. This may suggest that PVP and PVA are effective stabilizers only toward PANI-ES, due to the proposed multiple synchronized interactions with PANI. When PANI-ES is transformed in emeraldine base, this modification is accompanied by a change in the nature and extent of interactions with suspending polymer and morphology destabilization occurs. As expected, this process cannot be reverted: as it has been observed for FTIR spectra when $\mathrm{pH}$ is then brought back to acidic from alkaline, the colloidal dispersion with the original nanoscalar morphology is not restored.

The influence of dilution and $\mathrm{pH}$ has been investigated further for CT-PANI and PVA-PANI in the light of their emissive properties that will be discussed in the following. The minimum chitosan content in the suspending medium to prevent macroscopic precipitation of polyaniline has been experimentally determined to be about 0.2 wt.\%. Therefore, CT-PANI dispersions have been further diluted 500 times with $0.2 \mathrm{wt} . \% \mathrm{CT} / \mathrm{AcA}$, the $\mathrm{pH}$ of these dispersions resulting about 4 . The $\mathrm{pH}$ was then brought to 2 and 6 by adding either $\mathrm{HCl}$ or $\mathrm{NaOH}$ to the aqueous chitosan/AcA solution used to dilute the original dispersions. At $\mathrm{pH} 2$ the rice-grain nanoparticulate morphology of CT-PANI is clearly evident (Fig. 6a), at pH 4 PANI nanoparticles are the smallest in size (5-20 nm) (Fig. 6) and at $\mathrm{pH} 6$ coalescence of particles yields an almost continuous film covering the TEM microscopy grid (Fig. 6f). At high magnification, PANI nanoparticles in CT-PANI also reveal quite distinct aligned crystal planes (Fig. 6a'), that are not evident in the pure CT deposit. At the same dilution, PVA-PANI deposited on the graphite stubs present a distinct globular morphology (Fig. 6d). In this case the aligned higher electronic density planes show a "hay bale" organization (Fig. 6d"). Morphological evidence of locally ordered structures has not been observed for PVP-PANI, with PVP being the only fully amorphous polymer among the three. This evidence further supports the assumption that the supramolecular organization of the polymeric surfactant used in PANI dispersion polymerization controls PANI chains organization and dynamics. 

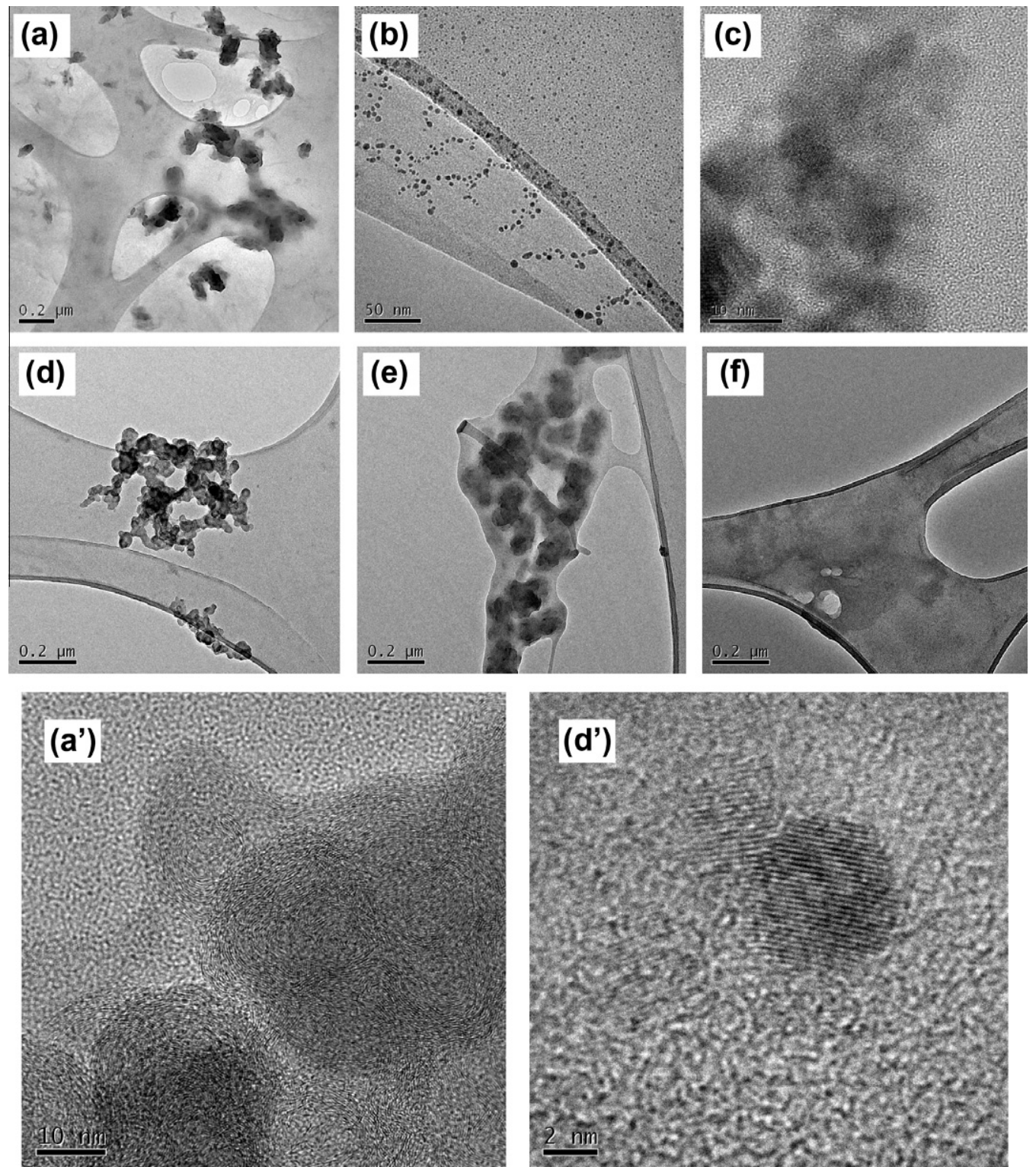

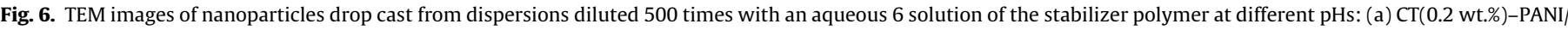

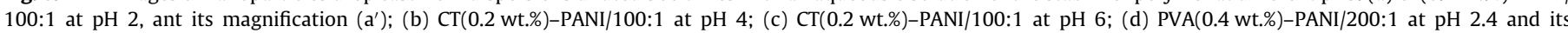
magnification (d'); (e) PVA(0.4 wt.\%)-PANI/200:1 at pH 5; (f) PVA(0.4 wt.\%)-PANI/200:1 at pH 9.

\subsection{Electrical properties}

While a comprehensive study of the electrical properties of the solid films obtained from dispersions at the variance of $\mathrm{pH}$ and drying conditions will be the subject of a dedicated paper, Impedance Spectroscopy has been applied here to both PVA-PANI and CT-PANI nanocomposite films prepared from the dispersions after dialysis at $\mathrm{pH} 1$ in order to determine the bulk conductivity of the nanocomposite material [42,21]. According to this approach, the complex dielectric function can be calculated as:

$\varepsilon^{*}(\omega)=\frac{Y^{*}(\omega)}{i \omega C_{0}}=\frac{\mathrm{C}}{C_{0}}-\mathrm{i} \frac{\mathrm{G}}{\omega C_{0}}=\varepsilon^{\prime}(\omega)-\mathrm{i} \varepsilon^{\prime \prime}(\omega)$

where $Y^{*}=[Z *(\omega)]-1$ is the measured complex admittance, $C$ and $G$ the measured capacitance and conductance, $\omega$ the angular frequency $(\omega=2 \pi f)$ and $C_{0}$ the equivalent capacitance of the free space. The frequency-dependent ac conductivity, $\sigma^{\prime}(\omega)$, is then obtained as: $\sigma^{\prime}(\omega)=\varepsilon^{\prime \prime}(\omega) \omega \varepsilon 0$

where $\varepsilon_{0}$ is the permittivity of free space and $\varepsilon^{\prime \prime}$ the imaginary part of complex permittivity. The values at low frequencies correspond to the dc conductivity, $\sigma_{d c}$, of the films. The difference in terms of bulk conductivity among the stabilizing polymer and the PANI nanocomposite film is dramatic. While the stabilizing polymer is an insulant material with a $\sigma^{\prime}(1 \mathrm{~Hz})$ of $\sim 10^{-10} \mathrm{~S} / \mathrm{m}$, the films formed by the corresponding PANI nanoparticles shows an increase of bulk conductivity (at $1 \mathrm{~Hz}$ ) of seven orders of magnitude. i.e. of $\sim 10^{-3} \mathrm{~S}$ / $\mathrm{m}$ for the nanocomposite films regardless the nature of the stabilizing polymer. This remarkable increase in conductivity would confirm that the synthesized PANI present in the film retains the middle oxidized emeraldine salt (ES) structure [43].

\subsection{Optical properties}

UV-Vis absorption and emissive properties of polyaniline have been studied varying the $\mathrm{pH}$ of the suspending medium. 


\subsubsection{UV-Vis absorption spectra}

In Fig. 7a UV-Vis absorption spectra of PVP-PANI, PVA-PANI and CT-PANI systems, tenfold diluted with the corresponding stabilizer aqueous solution, are shown. A more detailed investigation of the electronic spectra at the variance of $\mathrm{pH}$ has been also carried out for PVA-PANI and CT-PANI (Fig. 7b and c), in consideration of their emissive properties. It is worth pointing out that PVP aqueous solutions at all pHs do not show any significant absorption bands in the 350-900 nm region, while absorbance increases steadily below $350 \mathrm{~nm}$; PVA aqueous solutions at the same concentration have much lower absorbance and two weak bands at 276 and $320 \mathrm{~nm}$, that are attenuated at extreme pHs (inset of Fig. 7b);
chitosan/AcA aqueous solutions show intermediate absorbance values, increasing with $\mathrm{pH}$ in the investigated range (inset of Fig. 7c).

All PANI nanocolloids show similarities in their optical spectra: absorption peaks in the (i) 300-350 nm region and in the (ii) 550$800 \mathrm{~nm}$ region [44]. The $\mathrm{HCl}$ doped emeraldine salt $(\mathrm{pH} \sim 2)$ in PVP-PANI contributes with the characteristic absorption bands at about $350 \mathrm{~nm}$ in region (i), due to the $\pi-\pi^{*}$ transition of the reduced benzenoid repeat units, and at $790 \mathrm{~nm}$ in region (ii) for the presence of a polaron structure, respectively. For PVA-PANI and CT-PANI the $\pi-\pi^{*}$ transition is blue-shifted to $340 \mathrm{~nm}$, possibly due to the reduction in the average size of PANI NPs and a cor-
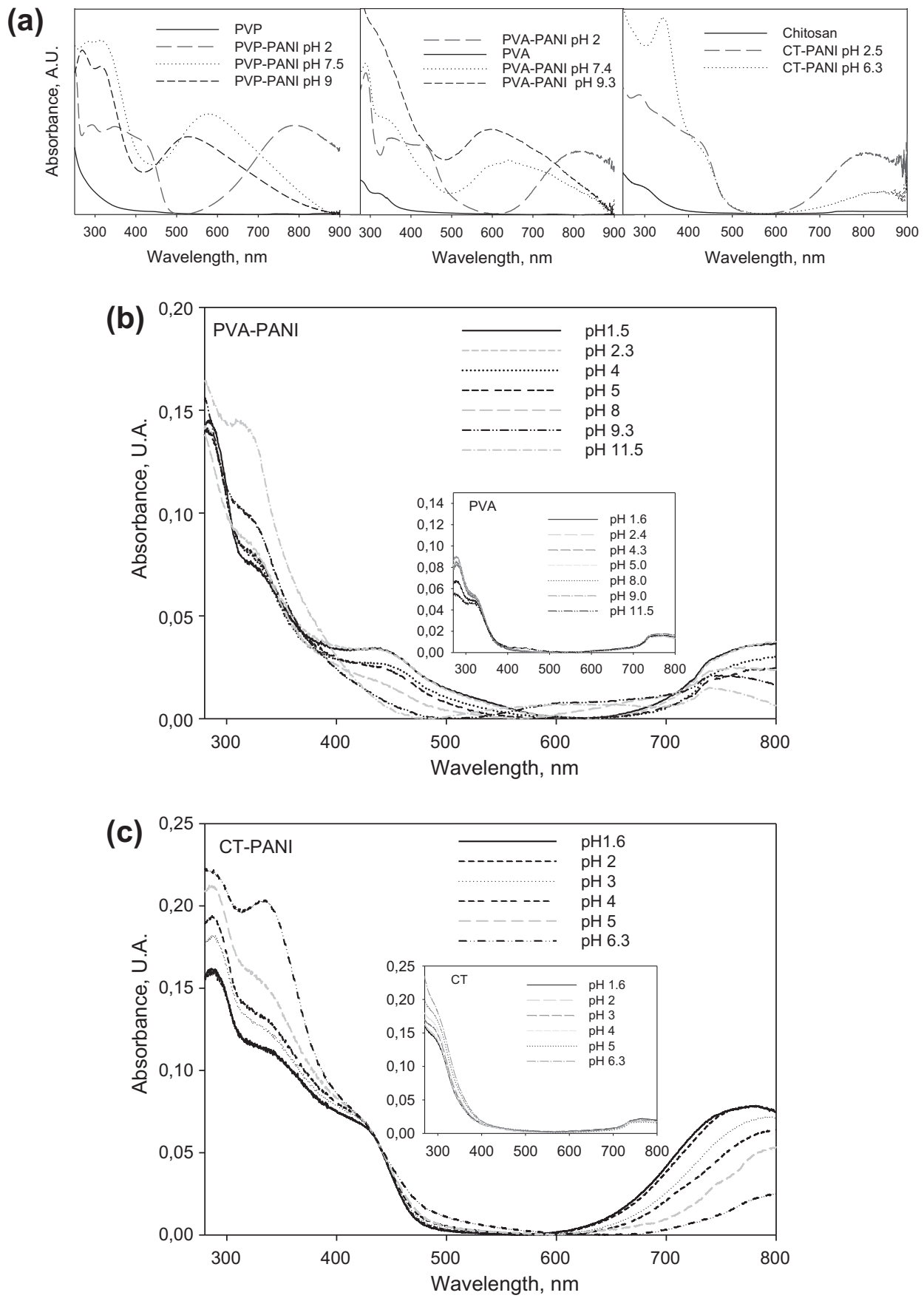

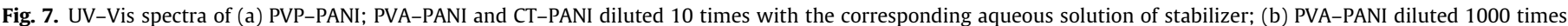

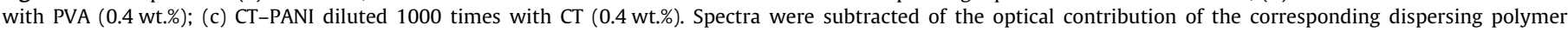
solutions. 
responding increase of band-gap. Moreover, the absorption in the visible range of the spectrum is significantly red-shifted to $810 \mathrm{~nm}$ and further, when $\mathrm{pH}$ is increasing from 2 to 5 (see Fig. $7 b$ and c). In general, this shift is associated to a free-carrier tail indicating that PANI chains are in an extended-chain conformation, in good agreement with TEM analysis. In addition, a third band at $\sim 430 \mathrm{~nm}$ is evident, attributable to the exciton transition caused by interband charge transfer from the benzenoid to the quinoid moieties in the protonated PANI (polaron/bipolaron transition) [44].

When $\mathrm{pH}$ approaches to 7, absorptions at $430 \mathrm{~nm}$ and $\sim 800 \mathrm{~nm}$ are no longer present, the peak in region (i) for PVP-PANI is considerably shifted down to $310-320 \mathrm{~nm}$ while it remains practically unmodified for PVA-PANI. A new broad band appears at about $580-650 \mathrm{~nm}$, attributable to the benzenoid to quinoid exciton transition (BQET). The value for the BQET is reported to be highly influenced by the specific arrangements of the polymer chains $[13,14,45-48]$. In particular, PVP-PANI shows a narrower and blue-shifted band than PVA-PANI. At the extreme pHs in the range $(\sim \mathrm{pH} 9)$ transformation of emeraldine base into pernigraniline base is occurring only when polyaniline is in the presence of PVP, as this brings the characteristic absorption in the visible range down to $\sim 530 \mathrm{~nm}$.

Finally, all systems show a distinct absorption band at $290 \mathrm{~nm}$ of decreasing importance with increasing the $\mathrm{pH}$. This peak has
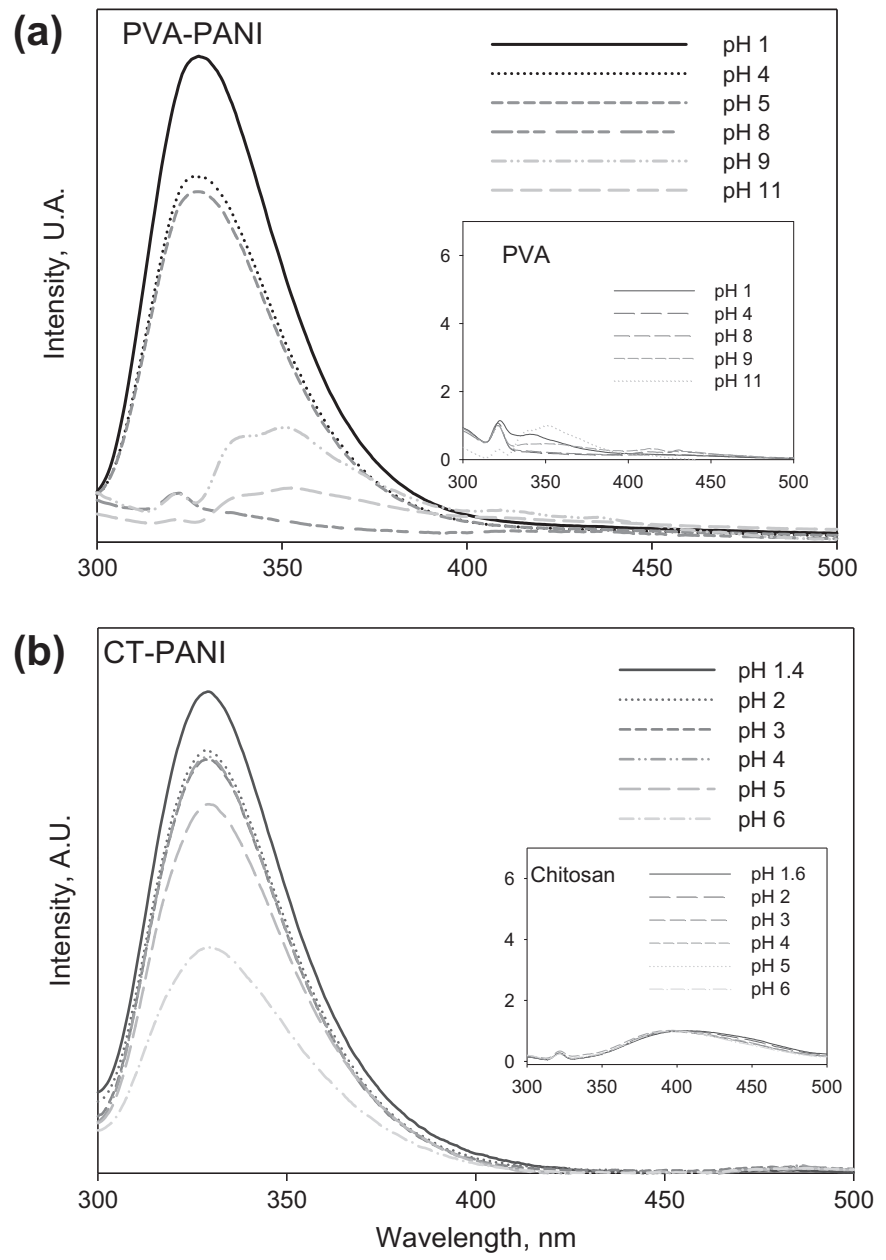

Fig. 8. Fluorescence spectra (excitation at $290 \mathrm{~nm}$ ) of 1000 dilute aqueous dispersions of nanoparticles (a) PVA-PANI (inset: PVA aqueous solution); (b) CTPANI (inset: chitosan aqueous solution). Spectra were normalized for the absorbance values at the corresponding excitation wavelength. been already observed by in situ UV-Vis measurements during the electrochemical polymerization of p-aminodiphenyl amine (p-ADPA) and attributed to an early intermediate formed in aniline electrochemical polymerization, when highly anodic potentials are reached in a pre-cycle run at high scan speed [49]. This highly soluble precursor origins from head-to-tail aniline dimerization and it acts as nucleus for further aniline polymerization. The reduced radical cations of p-ADPA, by symproportionizing with the oxidized form N-phenyl-quinonediimine, react with each other to give the tetrameric form. Aniline monomer units are able to attack these tetrameric polymer nuclei to grow the polymer chain.

Transposing this description to dispersion polymerization, we may speculate that the stabilizing polymer is adsorbing the tetrameric and higher oligomers once formed, thus shifting the symproportionizing equilibrium toward the reduced form. The PANI nanoparticles would grow fixing reduced benzenoid repeat unit sequences at the PANI-surfactant interface, whose presence also justifies the observed photoemissive behavior that will be discussed in the following. This aspect will be further investigated.

Indeed, FTIR carried out on polyaniline nanoparticles at acidic $\mathrm{pH}$ does not show any significant evidence of the quinonoid structures. Morphology destabilization is governed by a diminished interfacial interaction between the stabilizing polymer and PANI, therefore it is accompanied by a re-equilibration of the redox forms and by consequent electronic and conformational rearrangements that cause diminution or disappearance of the absorption band at
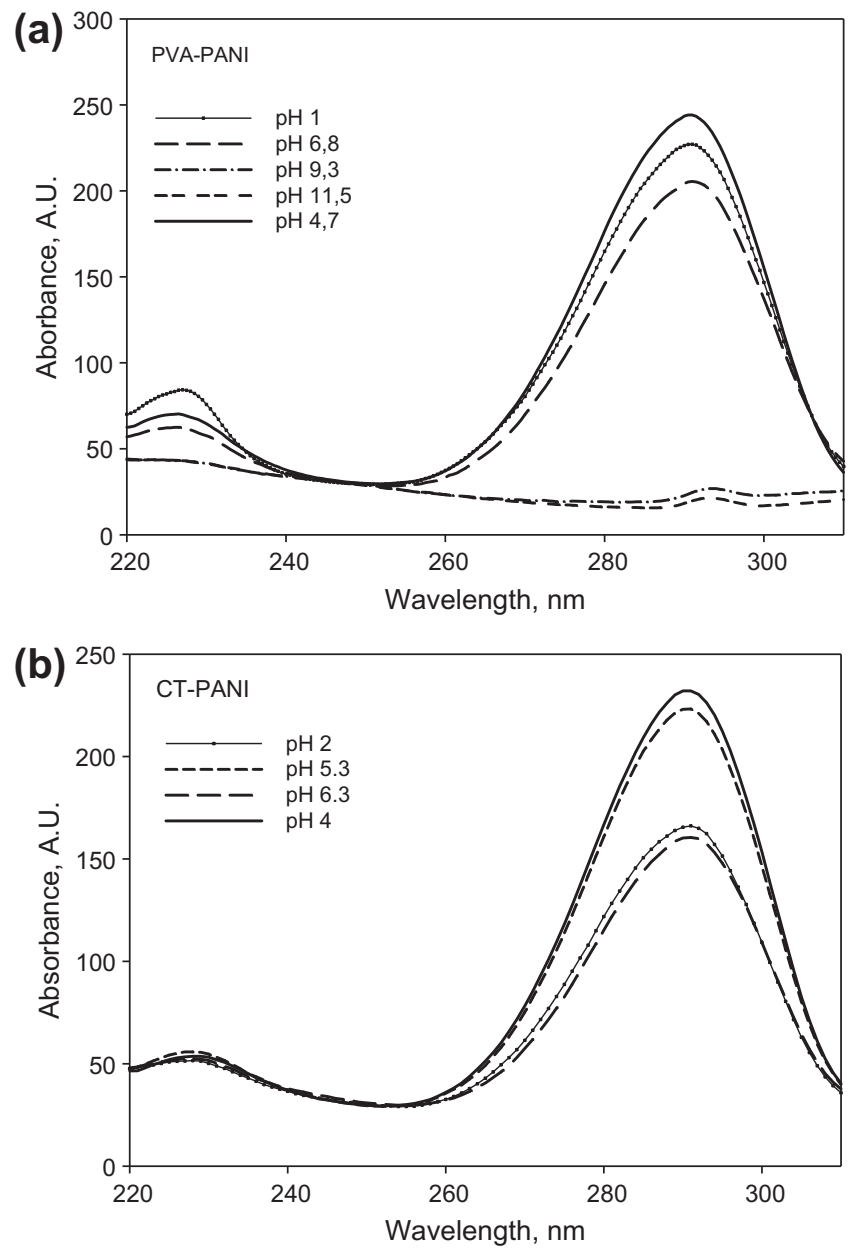

Fig. 9. Photoluminescence excitation (PLE) spectra of 1000 dilute aqueous dispersions of nanoparticles (a) PVA-PANI; (b) CT-PANI. 
$290 \mathrm{~nm}$ observed at $\mathrm{pH} 6.3$ for CT-PANI and at $\mathrm{pH}>7$ for PVAPANI (see Fig. 7c and b, respectively).

\subsubsection{UV-Vis luminescence spectra}

Both PVA-PANI and CT-PANI nanoparticles at acid pH show a distinct emission band at $320 \mathrm{~nm}$ when excited at $290 \mathrm{~nm}$, as displayed in Fig. 8a and b.

The two stabilizers do not present the above described emission band when excited a $290 \mathrm{~nm}$, as shown in the insets of Fig. 8. When $\mathrm{pH}$ of the dispersions is raised the emission intensity gradually decreases to disappear at $\mathrm{pH} 7$ and above. Interestingly, shifting the $\mathrm{pH}$ back to acidic does not restore the emissive properties as it did not give back the original nanoparticulate morphology. PVPPANI dispersions do not show emissive properties at any excitation wavelength and $\mathrm{pH}$.

Photoluminescence excitation (PLE) spectra in the UV-Vis range have been also collected at the variance of $\mathrm{pH}$ and wavelength of excitation for all systems. Fig. 9a and b shows a distinct absorption peak at $290 \mathrm{~nm}$ in the PLE spectra of the emissive systems. This evidence is confirming the role of PANI in the emissive properties of the corresponding colloids.

\section{Conclusions}

Nanoparticulate morphology stabilization/destabilization is at the basis of the $\mathrm{pH}$ dependent emissive behavior. The role of the stabilizing polymer is key in this respect. When it is able to support a genuinely nanoscalar dispersion of PANI-ES and it is strongly interacting with it, a "new functionality" is imparted to the particles through fixation of "phase segregated" sequences of reduced benzenoid units. While intrinsic luminescence of PANI NPs can be usefully exploited in applications, the dynamic organization of PANI chains in colloidal systems is an important aspect to be carefully controlled in any post-polymerization processes of PANI.

\section{Acknowledgements}

The work has been financially supported by University of Palermo (ex 60\% 2007). Dynamic light scattering measurements have been carried out at the CNR-IBF UO Palermo, with kind permission of Dr. D. Bulone. Helpful discussions with Dr. D. Bulone, Prof. S. Piazza and Prof. M. Leone are gratefully acknowledged.

\section{References}

[1] E.T. Kang, K.G. Neoh, K.L. Tan, Prog. Polym. Sci. 23 (1998) 277-324.

[2] G.G. Wallace, G.M. Spinks, L.A.P. Kane-Maguire, P.R. Teasdale, in: Conductive Electroactive Polymers. Intelligent Polymer Systems, Chapt. 5: Properties of Polyanilines, 3rd ed. CRC Press, Boca Raton, Florida, 2009, pp 179-186.

[3] H. Kuzumany, M. Bartonek, Europhys. Lett. 12 (2) (1990) 167-172.

[4] V. Saxena, B.D. Malhotra, Curr. Appl. Phys. 3 (2003) 293-305.

[5] A. Pron, P. Rannou, Prog. Polym. Sci. 27 (2002) 135-190.
[6] A. Guiseppi-Elie, Biomaterials 31 (2010) 2701-2716.

[7] H. Shi, J.I. Yeh, Nanomedicine 2 (5) (2007) 587-598.

[8] G. Wallace, G. Spinks, Soft. Matter. 3 (2007) 665-671.

[9] K. Lee, S. Cho, S.H. Park, A.J. Heeger, C. Lee, S. Lee, Nat. Lett. 14/4 (2006) 65-68.

[10] S.-H. Lee, D.-H. Lee, K. Lee, C.-W. Lee, Adv. Funct. Mater. 15 (2005) 1495-1500.

[11] M. Wan, J. Yang, J. Appl. Polym. Sci. 55 (1995) 399-405.

[12] M.K. Ram, G. Mascetti, S. Paddeu, E. Maccioni, C. Nicolini, Synth. Met. 89 (1997) 63-69.

[13] Y. Shimano, A.G. MacDiarmid, Synth. Met. 123 (2001) 251-262.

[14] S.-A. Chen, K.-R. Chuang, C.-I. Chao, H.-T. Lee, Synth. Met. 82 (1996) 207-210.

[15] J. Gong, J. Yu, Y. Chen, L. Qu, Mater. Lett. 57 (2002) 765-770.

[16] J. Gong, X.J. Cui, Z.W. Xie, S.G. Wang, L.Y. Qu, Synth. Met. 129 (2002) 187-192.

[17] F. Dierschke, J. Jacob, K. Mullen, Synth. Met. 156 (2006) 433-443.

[18] M. Amrithesh, S. Aravind, S. Jayalekshmi, R.S. Jayasree, J. Alloys Comp. 449 (2008) 176-179.

[19] M. Wohlgenannt, Z.V. Vardeny, J. Phys: Condens. Matter. 15 (2003) 83-107.

[20] C. Dispenza, M. Leone, C. Lo Presti, F. Librizzi, G. Spadaro, V. Vetri, J. Non-Cryst. Solids 352 (2006) 3835-3840.

[21] C. Dispenza, C. Lo Presti, C. Belfiore, G. Spadaro, S. Piazza, Polymer 47 (2006) 961-971.

[22] C. Dispenza, G. Fiandaca, C. Lo Presti, S. Piazza, G. Spadaro, Radiat. Phys. Chem. 76 (2007) 1371-1375.

[23] P. Stepanek, Data analysis in dynamic light scattering, in: W. Brown (Ed.), Dynamic Light Scattering, Oxford University, Oxford, UK, 1993, pp. 177-240.

[24] N.V. Blinova, J. Stejskal, M. Trchová, J. Prokes, M. Omastová, Eur. Polym. J. 43 (2007) 2331-2341.

[25] C.-H. Chen, J. Appl. Polym. Sci. 89 (2003) 2142-2148.

[26] J. Stejskal, I. Sapurina, M. Trchová, E.N. Konyushenko, Macromolecules 41 (2008) 3530-3536.

[27] I. Sěděnková, M. Trchová, J. Stejskal, Polym. Degr. Stab. 93 (2008) 2147-2157 (and references herein).

[28] S. Goel, A. Gupta, K.P. Singh, R. Mehrotra, H.C. Kandpal, Mater. Sci. Eng. A 443 (2007) 71-76.

[29] I. Sapurina, J. Stejskal, J. Coll. Interf. Sci. 274 (2004) 489-495.

[30] G.G. Wallace, G.M. Spinks, L.A.P. Kane-Maguire, P.R. Teasdale, in: Conductive Electroactive Polymers. Intelligent Polymer Systems, Chapt. 4: Synthesis of Polyanilines, 3rd ed. CRC Press, Boca Raton, Florida, 2009, pp. 137-178.

[31] C. Dearmitt, S.P. Armes, J. Coll. Interf. Sci. 150 (1) (1992) 134-142.

[32] X.H. Xu, G.L. Ren, J. Cheng, Q. Liu, D.G. Li, J. Mat. Sci. 41 (2006) 3147-3149.

[33] D. Chattopadhyay, B.M. Mandal, Langmuir 12 (1996) 1585-1588.

[34] S.E. Moulton, P.C. Innis, L.A.P. Kane-Maguire, O. Ngamna, G.G. Wallace, Curr. Appl. Phy. 4 (2-4) (2004) 402-406.

[35] J. Pecher, S. Mecking, Chem. Rev. 110 (2010) 6260-6279.

[36] C. Dispenza, M. Ricca, C. LoPresti, G. Battaglia, M. La Valle, D. Giacomazza, D. Bulone, Polym. Chem. 2 (2011) 192-202.

[37] R. Murugesan, G. Anitha, E. Subramanian, Mater. Chem. Phys. 85 (2004) 184194.

[38] P.-D. Hong, C.-M. Chou, C.-H. He, Polymer 41 (2001) 6105-6112.

[39] J. JanČa, D. Moinard, E. JanČová, N. Gospodinova, Int. J. Polym. Anal. Charact. 6 (2000) 213-228

[40] F. Chen, P. Liu, AIChE J. 57 (3) (2011) 599-605.

[41] G. Lamarque, J.-M. Lucas, C. E Viton, A. Domard, Biomacromolecules 6 (2005) 131-142.

[42] C.D. Hurd, M.F. Dull, K.E. Martin, J. Am. Chem. Soc. 54 (5) (1932) 1974-1976.

[43] E. Barsoukov, J.R. Macdonald, in: Impedance spectroscopy: theory, experiment, and applications, Wiley, Hoboken, N.J., New York, 2005.

[44] M.S. Cho, S.Y. Park, J.Y. Hwang, H.J. Choi, Mat. Sci. Eng. C 24 (2004) 15-18.

[45] P.M. McManus, S.C. Yang, R.J. Cushman, J. Chem. Soc. Chem. Comm. 1556 (1985).

[46] O.P. Dimitriev, Synth. Met. 125 (2002) 359-363.

[47] J.E. Albuquerque, L.H.C. Mattoso, D.T. Balogh, R.M. Faria, J.G. Masters, A.G. MacDiarmid, Synth. Met. 113 (2000) 19-22.

[48] J.E. Albuquerque, L.H.C. Mattoso, R.M. Faria, J.G. Masters, A.G. MacDiarmid, Synth. Met. 146 (2004) 1-10.

[49] A. Zimmermann, U. Ktinzelmann, L. Dunsch, Synth. Met. 93 (1998) 17-25. 